$3-31-2017$

\title{
Find Me If You Can: Mobile GPS Mapping Applications Forensic Analysis \& SNAVP the Open Source, Modular, Extensible Parser
}

Jason Moore

Ibrahim Baggili

University of New Haven

Frank Breitinger

Follow this and additional works at: https://commons.erau.edu/jdfsl

Part of the Computer Engineering Commons, Computer Law Commons, Electrical and Computer Engineering Commons, Forensic Science and Technology Commons, and the Information Security Commons

\section{Recommended Citation}

Moore, Jason; Baggili, Ibrahim; and Breitinger, Frank (2017) "Find Me If You Can: Mobile GPS Mapping Applications Forensic Analysis \& SNAVP the Open Source, Modular, Extensible Parser," Journal of Digital Forensics, Security and Law. Vol. 12 , Article 7. DOI: https://doi.org/10.15394/jdfsl.2017.1414

Available at: https://commons.erau.edu/jdfsl/vol12/iss1/7

This Article is brought to you for free and open access by

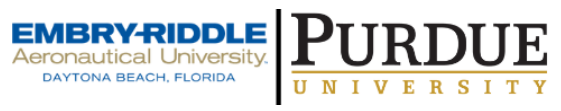
the Journals at Scholarly Commons. It has been accepted for inclusion in Journal of Digital Forensics, Security and Law by an authorized administrator of (c)ADFSL Scholarly Commons. For more information, please contact commons@erau.edu. 


\title{
FIND ME IF YOU CAN: MOBILE GPS MAPPING APPLICATIONS FORENSIC ANALYSIS \& SNAVP THE OPEN SOURCE, MODULAR, EXTENSIBLE PARSER
}

\author{
Jason Moore, Ibrahim Baggili and Frank Breitinger \\ Cyber Forensics Research and Education Group (UNHcFREG) \\ Tagliatela College of Engineering \\ University of New Haven, West Haven CT, 06516, United States \\ e-Mail: moore.p.jason@gmail.com, \{IBaggili, FBreitinger $\} @$ newhaven.edu
}

\begin{abstract}
The use of smartphones as navigation devices has become more prevalent. The ubiquity of handheld navigation devices such as Garmins or Toms Toms has been falling whereas the ownership of smartphones and their adoption as GPS devices is growing. This work provides a comprehensive study of the most popular smartphone mapping applications, namely Google Maps, Apple Maps, Waze, MapQuest, Bing, and Scout, on both Android and iOS. It details what data was found, where it was found, and how it was acquired for each application. Based on the findings, the work allowed for the construction of a tool capable of parsing the data from all of the aforementioned applications as well as creating maps of the locations attained. It was discovered that much data relating to the user's navigation history, be it addresses, latitude longitude points, etc., were stored on the user's device. It was also found that in almost all cases, discerning whether the user had actually traveled to a destination from the mapping application data was not possible.
\end{abstract}

Keywords: Mapping application forensics, iOS forensics, Android forensics, GPS forensics, Waze forensics, Google Maps forensics, Apple Maps forensics, MapQuest forensics, Scout forensics, Bing forensics.

\section{INTRODUCTION}

In 2015, global smartphone ownership reached 1.859 billion people. This is expected to rise to over 2 billion by the end of 2016 , and by the end of 2018 smartphone penetration is projected to increase to just over $36 \%$ of the global population (2.48 billion users) (Statista, 2016). Smartphones have surpassed the ownership level of personal computers and have become a main element of crime scene investigations (Umale, Deshmukh, \& Tambhakhe, 2014). McMillan, Glisson, and Bromby (2013) conducted research in the United Kingdom which illustrated the rise of criminal activities involving smartphones at an average rate of growth of ten cases per year from 2006 to 2011.

A segment of applications that has gone largely unnoticed by the research community is that of mapping applications. Paralleled with the increase in smartphone adoption as navigation devices has been the decrease in the use of hand-held navigation devices like Garmins (Statista, 2012). In the United States alone, Google Maps and Apple Maps, have 64.5 and 42 million users, respectively (Buczkowski, 2014), while the remaining applications seen in Table 1 account for around a total of 50 million users (Cohan, 2013). Worldwide, both Google Maps (46.7\%) and Apple Maps (26.2\%) have signifi- 
cant penetration in the mobile domain (Statista, 2015).

The nature of mapping applications, navigating a user to a destination, directing users around traffic, etc., makes them likely to hold data on where the user has been, what time they were there, their traveling tendencies, etc., some even offer chatting services with other users (see Table 2). It is apparent why data such as this may be of importance to investigators and as such is the reason for the examination of the highly adopted mapping applications listed in Table 1.

Table 1: Tested applications

\begin{tabular}{lll}
\hline Application & Android Vers. & iOS Vers. \\
\hline Google Maps & 9.6 .1 & 4.8 .62649 \\
Apple Maps & NA & 8.3 \\
Waze & 3.9 .3 .0 & 3.2 .9 .1 \\
MapQuest & 2.7 .7 & 4.8 \\
Bing Maps & 5.2 .0 .20140710 & 5.4 \\
Scout GPS & 2.5 .1 .0021 & 2.1 .1 \\
\hline
\end{tabular}

At the time of writing this paper, literature on the forensics of smartphone mapping applications was sparse. Our work aimed at filling the gap in the scientific literature.

Our research informs practitioners, researchers and the digital forensics community at large on methods of extracting data from the applications in Table 2. It identified the digital forensic artifacts that can be found by analyzing each of these applications and resulted in a tool that can be used to analyze potential digital evidence from the applications.

Manual retrieval of data from these applications would take a considerable amount of time and expertise. Not all of the files associated with these applications can be opened and read by human eyes. Some files need to be decoded, some have no structure making them difficult to follow, and certain files contain data of interest which is hidden amongst an immense amount of irrelevant data.

Smart NAVigation Parser (SNAVP), the tool constructed during this research (see Sec. 5), combats all of these issues by i) outputting an organized, searchable, sortable, and easy to read Excel file where all of the data related to these applications can be found ii) eliminating the need to have a person with the expertise needed to decode/translate certain files to a human readable format, and iii) removing the need for an investigator to manually search through a device's forensic image to locate and extract evidence. The tool also contributes a framework to the digital forensics community that can easily be built upon to include new versions of the tested applications as well as any new mapping applications released in the future.

In the remainder of the paper we first discuss related work in Sec. 2. We then describe our methodology in Sec. 3. We later share our findings of the artifacts found from the various mapping applications in Sec. 4. The constructed extensible SNAVP tool is then described in Sec. 5 . We lastly present future work and limitations in Sec. 6 and conclusions in Sec. 7.

\section{RELATED WORK}

\subsection{Hand-held satellite navigation devices}

Research has been conducted in the area of hand-held satellite navigation devices such as Garmins, TomToms, and Magellans (Van Eijk \& Roeloffs, 2010; Arbelet, 2014). Past work illustrated the importance of the digital evidence that could be extracted from these devices such as where the user has been, how they got there, who owns them, favorite addresses, etc. (Last, 2009). Evidence such as this may lead to a number of important discoveries during investigations including behavioral profiles of those who have used the device (Colombini, Colella, Castiglione, \& Scognamiglio, 2012).

Notwithstanding, satellite navigation devices are being replaced by smartphone mapping applications. Garmin reported a company worth less than a third of its value in 2007 as well a 15 percent reduction in sales in 2013 (Leber, 2013). Much of this drop was accredited to the increasing ubiquity of smartphones and the usage of the mapping applications on them replacing Global Positioning System (GPS) devices. 
Table 2: Application features

\begin{tabular}{lccccccc}
\hline Application & Navigation & Directions & Chatting & ETA & Home/Work Address & Favorites & Location Sharing \\
\hline Google Maps & $\checkmark$ & $\checkmark$ & - & - & $\checkmark$ & $\checkmark$ & - \\
Apple Maps & $\checkmark$ & $\checkmark$ & - & - & - & $\checkmark$ & - \\
Waze & $\checkmark$ & $\checkmark$ & $\checkmark$ & $\checkmark$ & $\checkmark$ & - & $\checkmark$ \\
MapQuest & $\checkmark$ & $\checkmark$ & - & - & $\checkmark$ & - & - \\
Bing Maps & - & $\checkmark$ & - & - & - & - & - \\
Scout GPS & $\checkmark$ & $\checkmark$ & $\checkmark$ & $\checkmark$ & $\checkmark$ & $\checkmark$ & - \\
\hline
\end{tabular}

\subsection{Mobile phone forensics}

McMillan et al. (2013) conducted a survey which found that calls and Short Message Service (SMS) data held the most evidential importance during investigations, however, user and application data were also found to be of high importance. Much of the work in the mobile forensics domain has focused on discovering these types of data on various Operating Systems (Hoog, 2011; Casey, Bann, \& Doyle, 2010; Simão, Sícoli, Melo, Deus, \& Sousa Júnior, 2011).

For instance, Al Mutawa, Baggili, and Marrington (2012) investigated the forensic artifacts that could be extracted from different social media applications such as Facebook, Twitter and MySpace. Furthermore, researchers such as Mahajan, Dahiya, and Sanghvi (2013) and Thing, Ng, and Chang (2010) examined what digital forensic artifacts could be recovered from different messaging services and applications such as WhatsApp and Viber. Newer studies focused on examining the network forensic implications of similar social-messaging applications (Karpisek, Baggili, \& Breitinger, 2015; Walnycky, Baggili, Marrington, Moore, \& Breitinger, 2015).

Maus, Höfken, and Schuba (2011) conducted a study concerning geodata on Android phones where they used geodata from all applications on a device which stored location data in an attempt to provide the investigator with a viewable map of where the user had been.

However, retrieving data from mobile devices has comes with several challenges for researchers and investigators (Bennett, 2012). The nature of smartphones, the Central Processing
Unit (CPU) architecture, their processing and memory resources, as well as the sheer number of used Operating Systems (OS), severely complicates the forensic acquisition and analysis of these devices (Barmpatsalou, Damopoulos, Kambourakis, \& Katos, 2013).

The process gets more complex when one takes into account the high rate of change in the mobile field (new applications, application versions, OS versions, etc.), the number of different devices that are in use, and the lack of software, hardware and interface standardization (Lessard \& Kessler, 2010; Baggili, Mislan, \& Rogers, 2007). Casey and Stellatos (2008) also noted that encryption of the data on these devices is becoming more prevalent having notable implications on digital forensic investigations. These challenges present investigators and researchers with a difficult task whenever a mobile device is being examined.

\subsubsection{Android forensics}

The challenges posed by Android devices stem from the wide array of versions the platform supports. Many developers and companies put their own twist on the platform when releasing their devices leading to every device/Android version combination to possess unique characteristics. A minor difference in the Android version may require extensive testing and validation of tools before they can be deemed forensically sound on a given system (Hoog, 2011).

Android forensics is an active area of research. For example, Vidas, Zhang, and Christin (2011) focused on developing a general collection methodology for the vast amount of Android devices. Distefano, Me, and Pace (2010), however, 
focused on discovering different anti-forensic methods used on Android devices. Albano, Castiglione, Cattaneo, and De Santis (2011) developed a technique that provided the ability to modify and erase, securely and selectively, digital evidence on an Android device using simple software tools that are commonly seen on *nixlike operating systems such as Android.

The analysis of phone memory has also been of importance to Android forensics as valuable data such as passwords, text messages, etc. may be contained within the memory (Thing et al., 2010). Many tools have been created to aid in this process.

Several plugins for Volatility - an advanced memory forensics framework - were created to read data such as passwords, chat messages, user names, and e-mail (Macht, 2013). Sylve, Case, Marziale, and Richard (2012) designed a kernel module for dumping memory that addressed the difficulties in developing device-independent acquisition tools while Leppert (2012) focused on acquiring and analyzing heap-dumps of Android applications.

\subsection{2 iOS forensics}

Breaking the security barriers of the newer versions of iOS devices so that physical images may be acquired without jailbreaking the device has become increasingly difficult (Belenko \& Sklyarov, 2011). Jailbreaking involves removing restrictions on the device so that a physical image may be acquired, however, this process alters data on the device itself possibly tainting its forensic value. One would have to know that none of the user data was altered and that the exact alteration of any other data on the device be known for an acquisition method to be considered forensically sound.

The difficulty of acquiring a physical image of an iOS device has made other extraction methods more prominent when dealing with newer iOS devices. A common approach leverages the iPhone backup files created with the iTunes backup utility (Bader \& Baggili, 2010; Morrissey \& Campbell, 2010). This method only acquires data that has been synchronized by the backup protocol, but as stated by Hoog and Strzempka
(2011) most of the allocated data can be retrieved using this method. This method was determined to be the premier method for logical acquisition for iOS devices (Tso, Wang, Huang, \& Wang, 2012). Another often used approach is logical extraction. This procedure extracts active folders and files directly from the iPhone, but does not retrieve any data from the unallocated space.

\section{METHODOLOGY}

The methodology used in this research followed the guidelines for forensically examining artifacts presented by NIST (Kent, Chevalier, Grance, \& Dang, 2006). The smartphones that were used in our examination were a Samsung S4 Active (SGH-i537) running Android version 4.4.2 and an iPhone 4s Model A1387 running iOS 8.2. Towards the end of the research, the Samsung S4 Active stopped turning on and could no longer be used, therefore, a Samsung Galaxy S4 Zoom (SM-C101) running Android version 4.4.2 was used when investigating Google Maps and Bing.

Our research methodology was broken into three phases data creation and acquisition (Sec. 3.1), data analysis (Sec. 3.2), and tool creation (Sec. 3.3). The high level methodology is portrayed in Fig. 1.

\subsection{Phase I: data creation and acquisition}

Several actions were performed on each application. These actions were chosen based on the features of each application shown in Table 2. Each action was recorded and logged so that any data on the mobile device resulting from the given action could be discerned.

The devices were acquired using .XRY ${ }^{1}$. .XRY is software which forensically extracts data from a variety of mobile devices. A device image was acquired after each entry shown in Table 3 . The Samsung S4 Active and the Samsung Galaxy S4 Zoom were physically acquired, while all of the images of the iPhone $4 \mathrm{~S}$ were logical due to physical acquisition of the iPhone $4 \mathrm{~s}$ not being sup-

\footnotetext{
${ }^{1}$ Micro Systemation AB (MSAB), https: //www.msab .com/, last accessed 2015-09-29
} 


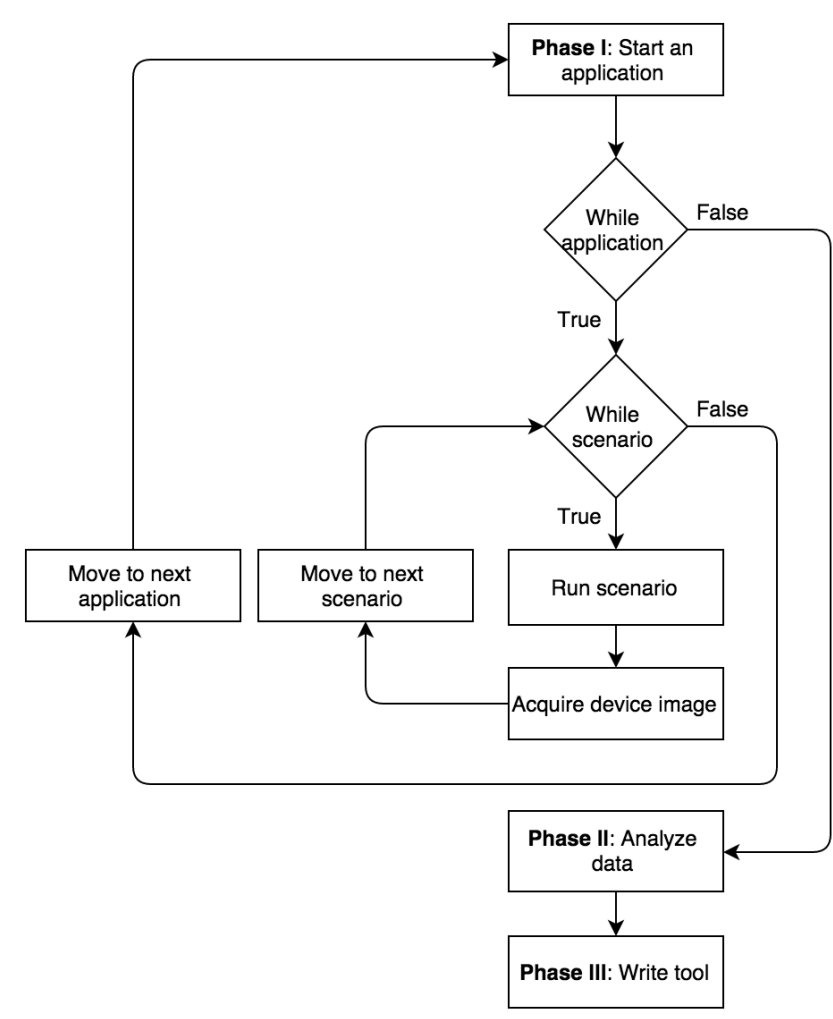

Figure 1: High level methodology for Android and $\mathrm{iOS}$

ported by any tools at the time of conducting the acquisition.

We note that although we used different acquisition methods on the devices, our work's scope focused on the allocated space, making the method of extraction not as significant as it would be had any of the data discovered was found in unallocated space.

\subsection{Phase II: data analysis}

Analysis of the acquired forensic images was completed using a variety of tools. .XRY and Cellebrite were used to mount and explore the acquired images to discover files related to the tested applications.

Once files were identified, several tools were utilized to inspect the file contents. WinHex ${ }^{2}$, a universal hexidecimal editor, was used to identify file headers and inspect the content of files that

\footnotetext{
${ }^{2}$ http://www.x-ways.net/winhex/, last accessed 2015-11-23
}

Table 3: Actions performed

\begin{tabular}{ll}
\hline$\#$ & Recorded action \\
\hline 1 & A fresh install of the application \\
2 & $\begin{array}{l}\text { A location was inserted into the appli- } \\
\text { cation and traveled to }\end{array}$ \\
3 & $\begin{array}{l}\text { A location was inserted into the appli- } \\
\text { cation and not traveled to }\end{array}$ \\
4 & $\begin{array}{l}\text { Application specific features such as } \\
\text { sending messages, sharing locations, } \\
\text { etc. }\end{array}$ \\
\hline
\end{tabular}

were not recognized by .XRY.

DB Browser for SQLite ${ }^{3}$ is a visual open source tool that allows for the creation, design, editing, and viewing of database files that are compatible with SQLite. This tool was used to explore the contents of any SQlite database encountered.

XML files were inspected with Sublime Text, a text editor for code, markup, and prose ${ }^{4}$, while any binary plist files encountered were inspected using a combination of Xcode 7 , a powerful IDE ${ }^{5}$, and the Terminal.

\subsection{Phase III: Smart Navigation Parser (SNAVP)}

SNAVP, the tool created during this research was designed to parse out as much of the data as possible that was found based on our findings. It was designed as a command line tool with modularity and extensiblity in mind so that additional parsers may be added it to it at any time. SNAVP is discussed in further detail in Sec. 5.

\section{RESULTS}

This section describes the results of the artifacts found in detail. For a summary of the artifacts found, refer to Table 5 and Table 6 .

\footnotetext{
${ }^{3}$ http://sqlitebrowser.org/, last accessed 2015-1209

${ }^{4}$ http://www.sublimetext.com/, last accessed 201512-07

${ }^{5}$ https://developer.apple.com/xcode/, last accessed 2015-11-20
} 


\subsection{Google Maps - Android}

Google Maps contained four files of evidentiary value: da_destination_history, search_history.db, com.google.android.apps.maps_preferences.xml, and DATA_SYNC_DATA.

da_destination_history was a SQLite database file that contained location data related to the starting and ending locations that the user selected to retrieve directions to. In order for data to be present in this database the user had to have selected navigate after entering in locations. search_history.db, another SQLite database, held the user's search data. This meant that navigation was not necessary for data to appear in this database, the data was entered as soon as the user searched for any location within Google Maps.

com.google.android.apps.maps_preferences.xml was an XML file that contained the user's preferences and more specifically the last user account name to use Google Maps.

$D A T A \_S Y N C$ DATA contained cloud data based on the user account that was using the application. So, for example, if a user had saved a search using a different device, that location would be found in this file so that it could be used on any device as long as the user was signed into Google Maps.

\subsection{Google Maps - iOS}

No data that related to the user's searches, navigation, or saved locations were found on the iPhone, however, the file com.google.Maps.plist did include the user's last known location along with what seemed to be other files of interest. In com.google.Maps.plist was a reference to a plist file called 1436530344726.plist. The name of the plist file, 1436530344726, was in the form of a Unix timestamp that converted to July 10, 8:12 AM EST, the time at which the last search occurred while using Google Maps.

\subsection{Apple Maps}

Only one file, GeoHistory.mapsdata, was found to contain data related to the user's actions in Apple Maps. GeoHistory.mapsdata was a plist file that held data pertaining to what the user had searched for within the application as well as
Yelp reviews for the given locations if applicable.

\subsection{Waze - Android}

The files waze_log.txt, user.db, tiles_nt.db, and $t t s . d b$ were found to hold data related to the user's activity while using the Waze application.

waze_log.txt contained an immense amount of data. The file was separated into active sessions which were defined as the time from when the user had opened the Waze application to when they closed out of it. Three different actions were found to cause a RoutingRequest within this file i) when a route was requested by the user ii) when GPS signal was lost and then found again during navigation, and iii) when rerouting occurred during navigation. A RoutingRequest contained a starting and ending location in the form of latitude and longitude points for the requested route, and the two lines directly above each RoutingRequest contained a timestamp and current location of the user.

$u s e r . d b$ was a SQLite database that contained a plethora of data including the destination the user had received directions to, the time at which the directions were requested, and the Estimated Time of Arrivals (ETAs) that were sent or received. It is critical to note that the data mentioned appeared in the database at the time navigation was selected within Waze; meaning that there was no way to discern whether the user had actually traveled to the destination form the data found in this database.

tiles_nt.db was a SQLite database that contained data which related to images of the pieces of maps that were needed for navigation sessions. An approach to relate the entries in this database to the many locations that were navigated to was not discovered and warrants future research.

$t t s . d b$ was another SQLite database. It held paths to the audio direction files that were used during navigation, i.e. turn right, continue straight, etc. However, a way to link the entries in this table to specific navigation sessions was not found.

Waze uniquely had the feature of sending and receiving messages to other users. The messages that were sent and received were not found on the device, however, prior research by Storozuk 
(2014) found that the messages could be found in the device's memory.

\subsection{Waze - iOS}

The only file found on the logical image of the iPhone was user.db. This SQLite database had the exact same structure and data as its Android counterpart (see Sec. 4.4).

\subsection{MapQuest - Android}

A SQLite database named search.db contained all of the data pertaining to the places that the user navigated to while using MapQuest. Three fields within the search table were found to hold the most importance in this research.

Two fields contained UNIX timestamps. The ctime field referred to when the user had searched for directions, while the atime field referred to the time that MapQuest actually retrieved the directions that were queried.

The third field was titled json and was a json field that contained the address and longitude and latitude coordinates of the destination along with a userInput key that held the text that the user had actually searched for.

\subsection{MapQuest - iOS}

The data that related to the user's navigation history while using MapQuest was found in the file com.aol.mapquest.plist. This plist file contained data such as latitude and longitude coordinates and addresses of destinations searched for or marked as a favorite by the user. However, there were no timestamps associated with these entries and the data structure was the same whether the destination was actually traveled to or not.

\subsection{Bing Maps}

Bing Maps was unique in that it was not a stand alone mapping application; the Bing Maps segment was just a part of the overall Bing application. As the purpose of this research was to test mapping applications, only the Bing Maps portion of the application was tested. Further, Bing had the option to search privately, and if the user was in private mode, Bing claimed that the users search history would not be stored by the application.

\subsubsection{Bing Maps - Android}

A single SQLite database file, SearchHistoryDatabase.db, held the user's search history when private browsing was not enabled. It had a simple structure of three tables with the table of interest being SearchHistory. The NAME field in this table referred to the text that was searched for and the $L A S T$ field correlated with the time that the search occurred.

\subsubsection{Bing Maps - iOS}

The iOS version of Bing was found to hold several files that contained data regarding the actions performed by the user, a History.plist file along with multiple ${ }^{*}$.dat files.

History.plist contained the search history of the user as long as private searching was not enabled. Any search that occurred while private searching was enabled did not result in an entry in Historys.plist. For an entry to be made here, the location had to be searched for using the search bar of the Bing Maps application. If the "get directions" icon was pressed prior to searching for a location then that location would not appear in Historys.plist.

The * ${ }^{*}$ dat files, where the ${ }^{*}$ represents an integer value, were found at two locations. The nonprivate version of the ${ }^{*}$. dat file was stored in the Documents/Journal directory. This directory did not exist until a search had occurred within the application, while the private version of these files were stored in the Documents/PriJournal directory, and similar to the non-private version, the PriJournal directory did not exist until a search had occurred when privacy was enabled.

It was found that the highest numbered ${ }^{*}$.dat file contained data on the most recent location that was searched by the user, with or without private browsing enabled.

\subsection{Scout - Android}

Two files were found which contained data deemed of evidentiary value, a SQLite database named scoutAppDatabase. $d b$ and an XML file titled Route_*.

scoutAppDatabase.db contained eight tables that held data pertaining to the user's actions while using Scout. This included members of 
chat sessions, the messages themselves, configuration data, the address along with the longitude and latitude coordinates of locations that were navigated to, and usernames of friends of the user.

A different Route $_{-}{ }^{*}$ file was created each time a location was navigated to where the ${ }^{*}$ was replaced by the UNIX timestamp of when the navigation session was first created. Within the file was detailed information about every route that was offered to the user for a given destination and also specified the exact route that the user had chosen. Each route was broken up into segments, thereby allowing one to reconstruct each given route by combining their respective segments.

Unfortunately, in both files, the location data was created at the time that navigation was requested, making it difficult to discern whether the user had actually traveled to the destination.

\subsection{Scout - iOS}

Only one plist file, namely com.telenav.scout.push store.log was found to hold data related to user's navigation, however, the data within this file was incomplete. For example, the file held the UNIX timestamp of when the navigation was started and only the final direction of the navigation session, i.e. in one of the test scenarios, the only direction found was "Turn left to Ruden St" (the final direction before the user would reach their actual destination). Because of this, the sequence of actions performed on the iOS device were completed several times as to ensure that the lack of data was not caused by an error, but still, only incomplete data was found in com.telenav.scout.pushstore.log.

Our findings helped us construct the basis for a parser for all of these applications which we discuss in the Sec. 5 that follows.

\section{SMART NAVIGATION PARSER (SNAVP)}

In its initial release, SNAVP was designed as a command line tool that took one required parameter and one optional parameter. The re- quired parameter was signified by a $-i$ and took the location of the input, i.e. a file, a directory, a device image, a zip file etc., as an argument. The optional parameter was signified by a $-o$ and was the location where the program would write its output to. If no argument was provided for the output location, the output defaulted to the current working directory.

After the input was received, the program proceeded to determine the nature of the input, i.e. whether it is a tar file, an image file, a single file, or a directory. The type of input determined the next steps, a tar file would have to be unpacked, an image would have have to be mounted, etc., however, we note that not all image files were mountable (see Sec. 6). Each file in the input was then iterated over and had its file header or extension checked to determine if it was a file of interest.

A file of interest was defined as a binary plist (bplist), a sqlite database, a text file, or an XML file. These were all of the file types from the tested applications that were found to hold digital evidence of forensic value. If a file was of interest, the parsers would run their validity check against the file, and if passed, the parser would then parse the file and send the parsed data back to the main program where it would be written out to an Excel file.

The first sheet of every Excel file, titled Parsers Ran (see Figure 2), lists the parsers that were run on the input given, provide the file path to the file that they were run on and the color of the marks for each respective application that can be seen on the All Applications Map (Figure 3). The data retrieved from each individual parser was contained on its own respective sheet within the created Excel document.

\subsection{Program structure}

The first file shown in Figure 4 is ParseControl.py. This file controlled the overall program. It received the user's input, ran the necessary parsers, collected the data, created the map files, and wrote it all out to an Excel file.

The Dependencies folder contained three key elements of SNAVP. FileCheck.py determined whether or not a given file was of the type txt, 


\begin{tabular}{l|ll|l|} 
Name & File Path & Color Key \\
\hline Google_Maps_Nav & /Users/Jay/Documents/ThesisTestZip/extracted/Thesis/da_destination_history & Orange \\
\hline Scout_1430235980 & /Users/Jay/Documents/ThesisTestZip/extracted/Thesis/Route_1430235980.xml & Copper \\
\hline Scout_Messages & /Users/Jay/Documents/ThesisTestZip/extracted/Thesis/scoutAppDatabase.db & NA \\
\hline Scout_Navigation & /Users/Jay/Documents/ThesisTestZip/extracted/Thesis/scoutAppDatabase.db & Violet \\
\hline Google_Maps_Search & /Users/Jay/Documents/ThesisTestZip/extracted/Thesis/search_history.db & Olive \\
\hline Apple_Maps & /Users/Jay/Documents/ThesisTestZip/extracted/Thesis/apple_maps/GeoHistory.mapsdata.log & NA \\
\hline Bing_Search & /Users/Jay/Documents/ThesisTestZip/extracted/Thesis/bing_android/SearchHistoryDatabase.db & NA \\
\hline Bing & /Users/Jay/Documents/ThesisTestZip/extracted/Thesis/bing_iOS/Historys.plist & NA \\
\hline MapQuest & /Users/Jay/Documents/ThesisTestZip/extracted/Thesis/mapquest_android/search2 & Light Blue \\
\hline Waze_Database & /Users/Jay/Documents/ThesisTestZip/extracted/Thesis/waze/user.db & Mustard \\
\hline Waze_Log & /Users/Jay/Documents/ThesisTestZip/extracted/Thesis/waze/waze_log.txt & Black \\
\hline
\end{tabular}

Figure 2: First sheet of SNAVP output

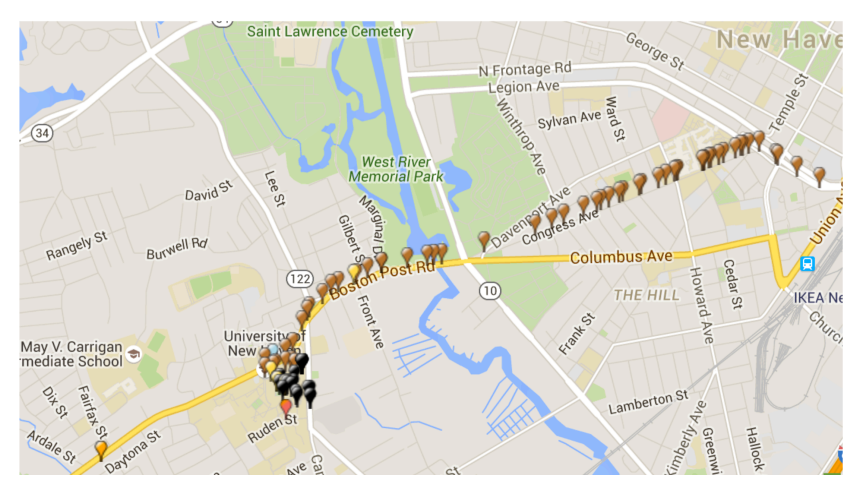

Figure 3: All applications map

sqlite, plist, or xml. This was determined by using the file header and/or the file extension. The action of Mount.py is determined by the input that was given. If the input was an image file, Mount.py would attempt to mount the file so that its contents could be read. If the input was a tar or zip file, it would extract the data to a given folder so that it may be read, etc. WorksheetData.py contained the WorksheetData class. This class was used to store the data from the individual parsers in a manner that Parse $C$ ontrol.py could interpret.

The Parsers folder contained all of the individual parsers that could be executed. As mentioned in Sec. 3.3, SNAVP was designed in a modular manner. To add a new parser to the tool, one would only have to add the parser file to this folder, and add the file name to the init.py file. As long as the parser created followed the WorksheetData class, it would be correctly inte-

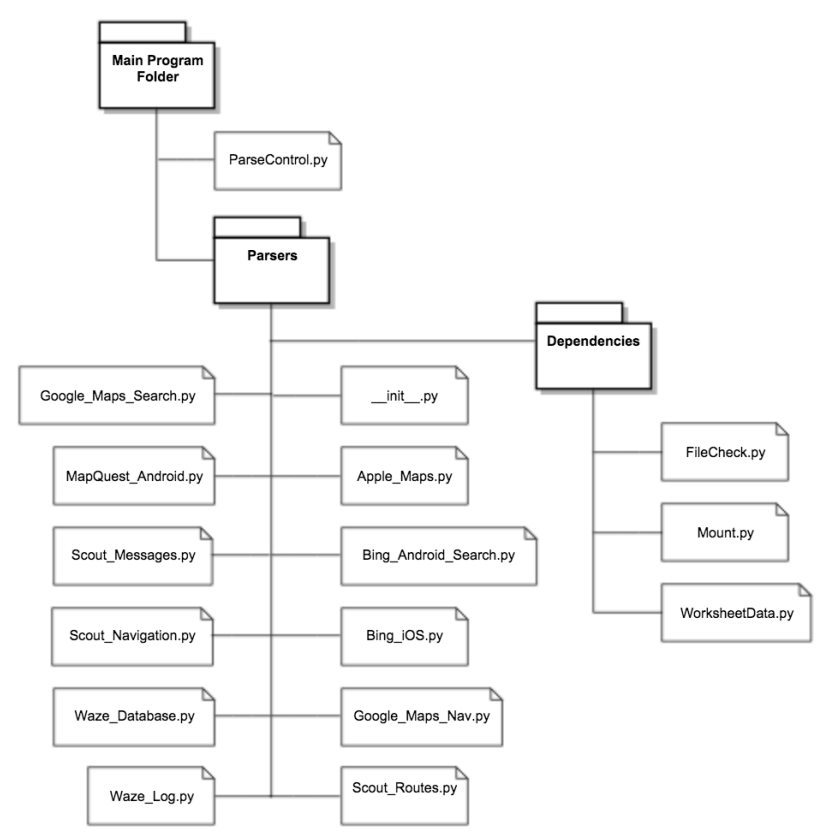

Figure 4: SNAVP structure

grated into SNAVP.

\subsection{Dependencies}

- The Sleuth Kit - is a C library along with a collection of command line tools that allow the analysis of disk images. Many tools, such as Autopsy, use The Sleuth Kit behind the scenes ${ }^{6}$. SNAVP used The Sleuth Kit to mount image files and extract files of in-

\footnotetext{
${ }^{6}$ The Sleuth Kit (TSK), http: //www.sleuthkit.org/, last accessed 2015-09-29
} 
terest so that they may be parsed.

- pytsk3 - is a Python package that is a Python binding for The Sleuth Kit ${ }^{7}$. This allowed SNAVP to programmatically mount images so that they could be searched to determine if any files of interest resided on the image. Those files were then extracted and parsed if applicable.

- pygmaps - is another Python package that is a Python wrapper for Google Maps ${ }^{8}$. This provided SNAVP with the capability to generate HTML files that plot given coordinates on Google Maps.

- ccl-bplist - is a Python module that helps deal with binary plist files (bplists) ${ }^{9}$. SNAVP utilized this module when attempting to parse any bplists that it encountered.

- XlsxWriter - is a Python module that allowed the creation of Excel XLSX files ${ }^{10}$. This module was used to organize and produce the parsed data into an Excel workbook in a meaningful manner.

\section{LIMITATIONS \& FUTURE WORK}

The rate at which new versions of applications are released is overwhelming. SNAVP was tested on the specific application versions shown in Table 1. This does not mean it will not work on other versions, however, it is possible that new and old versions of these applications have different file names, schemas for their files, etc. To combat this challenge SNAVP was created in a modular manner so that new parsers may be added to support any new or old versions of the tested applications as well as any additional GPS applications.

\footnotetext{
${ }^{7}$ pytsk3, https://github.com/py4n6/pytsk, last accessed 2015-09-29

${ }^{8}$ pygmaps, https://code.google.com/p/pygmaps/, last accessed 2015-09-29

${ }^{9}$ ccl-bplist, https://code.google.com/p/ ccl-bplist/, last accessed 2015-09-29

${ }^{10}$ XlsxWriter, http://xlsxwriter .readthedocs . org/, last accessed 2015-09-29
}

It is also important to note that the data that all of these applications were tested with was minimal in comparison to real data. The tool may error out on larger sets of data as not all possibilities could be accounted for with such small sets of test data.

When a device image file was used as input, not all of the images were mountable. For example, .XRY used a proprietary file type .xry that caused the program to error out when attempting to mount the image. Therefore, any image file that could not be mounted resulted in the user having to manually locate and extract the files of interest, then use the directory that the files were placed in as the input to the tool.

All of the latitude longitude points mapped by SNAVP were only accurate within the United States. Many of the applications did not put the decimal point in the latitude or longitude coordinate when it was saved; SNAVP had to perform this action. The decimal points vary in location depending on the actual latitude or longitude, therefore, the tool only guaranteed accuracy when it placed the decimal point for locations that were in the United States.

\section{CONCLUSION}

An immense amount of potential digital evidence related to the examined applications were discovered during this research (summarized in Table 5 and Table 6 ). This data could hold great evidentiary value during different types of investigations. The timestamps combined with the current locations in Waze can place users in certain vicinities at certain times thereby corroborating a story or an alibi. Knowing the route that a user had taken (data from the Scout application) can be used to locate evidence the suspect may have dropped along a route or locate cameras that may be used to track a suspect.

Table 4 rates the tested applications, 1 being highest and 10 being lowest, on the determined forensic value that they hold. The rankings are relative to each other and are a reflection on the amount and the type of data that was ascertained from each application during this research. It is important to note that none of the 
data mentioned in this work was found in unallocated space on the devices.

Table 4: Determined forensic value ranking of applications

\begin{tabular}{cl}
\hline Forensic value & Application \\
\hline 1 & Waze (Android) \\
2 & Scout (Android) \\
3 & Google Maps (Android) \\
4 & Waze (iOS) \\
5 & MapQuest (Android) \\
6 & Bing (iOS) \\
7 & MapQuest (iOS) \\
8 & Apple Maps \\
9 & Google Maps (iOS) \\
10 & Scout (iOS) \\
\hline
\end{tabular}

The Android version of Waze topped the list. In addition to the starting and ending navigational points, it was found to periodically store the current location of the user. Scout for Android was next, it was found to store all of the routes given to the user for a navigation session along with the route that was chosen by the user. It also contained messages that were sent between users on the application. Google Maps was ranked third because it stored the starting and ending location of provided routes. Only the top rated applications provided a starting location.

Applications ranked 4 through 6 were all similar as they provided data about the locations that were searched for or navigated to. Notwithstanding, they did not provide starting locations. MapQuest for iOS and Apple Maps were ranked $7^{\text {th }}$ and $8^{\text {th }}$ respectively. They both provided data on the user's search and navigation history but they did not store any timestamps with the data making it difficult to determine a timeline for the user. Google Maps for iOS ranked slightly ahead of Scout due to being able to determine the user's last location right before exiting Google Maps. The iOS version of Scout came in last as almost no data that related to the locations searched for or navigated to were found on the device. Note that the Android version of Bing is not listed in Table 4 since it could not be fully examined during this research.

The downfall to the data that was found on the devices was that actual travel was never required for data to be saved. Most of the tested applications stored their data when it was either searched for or navigated to. This could hurt cases as the user cannot be placed at a particular point or on a particular route without some other form of corroborating evidence. Again, the Android version of Waze was the only application that was found to store the user's current location at different times; this data was found in Waze's log file. To combat this challenge, future work could attempt to correlate GPS locations from multiple devices, as well as GPS log data from the OS with the ones found from the studied applications.

As mentioned in Sec. 2, valuable data was found on handheld navigation devices such as TomToms and Magellans. In fact, the data found was very similar to that which was found in this study. As with the data found on the smartphones, data such as locations entered to, home, work, and favorite addresses could be found on handheld navigation devices, however, there was one immense benefit gained from the data found on handheld navigation devices as opposed to the data found on smartphones. With handheld navigation devices one was able to differentiate whether a location in the GPS was actually traveled to (Nutter, 2008).

Although being able to differentiate between these two circumstances may be of importance, the rest of the mapping data found on the smartphones was decidedly similar to that found on handheld navigation devices. Thus, as it has been discussed by Strawn (2009) that GPS devices have helped prosecutors win cases, one may argue that the mapping data found on the smartphones during this research would still be of paramount importance during investigations.

\section{REFERENCES}

Albano, P., Castiglione, A., Cattaneo, G., \& De Santis, A. (2011). A novel anti-forensics technique for the android os. In Broadband and wireless computing, communica- 
tion and applications (bwcca), 2011 international conference on (pp. 380-385).

Al Mutawa, N., Baggili, I., \& Marrington, A. (2012). Forensic analysis of social networking applications on mobile devices. Digital Investigation, 9, S24-S33.

Arbelet, A. (2014). Garmin satnav forensic methods and artefacts: an exploratory study. (Unpublished doctoral dissertation). Edinburgh Napier University.

Bader, M., \& Baggili, I. (2010). iphone 3gs forensics: Logical analysis using apple itunes backup utility. Small scale digital device forensics journal, 4 (1), 1-15.

Baggili, I. M., Mislan, R., \& Rogers, M. (2007). Mobile phone forensics tool testing: A database driven approach. International Journal of Digital Evidence, 6(2), 168178.

Barmpatsalou, K., Damopoulos, D., Kambourakis, G., \& Katos, V. (2013). A critical review of 7 years of mobile device forensics. Digital Investigation, 10(4), 323-349.

Belenko, A., \& Sklyarov, D. (2011). Evolution of ios data protection and iphone forensics: from iphone os to ios 5. In Blackhat abu dhabi conference.

Bennett, D. (2012). The challenges facing computer forensics investigators in obtaining information from mobile devices for use in criminal investigations. Information Security Journal: A Global Perspective, 21(3), 159-168.

Buczkowski, A. (2014). The us mobile app report - google maps app 64.5m users, apple maps 42m - geoawesomeness. Retrieved 2015-9-29, from http://geoawesomeness.com/the-us -mobile-app-report-google-maps-app -64-5m-users-apple-maps-42m/

Casey, E., Bann, M., \& Doyle, J. (2010). Introduction to windows mobile forensics. digital investigation, 6(3), 136-146.

Casey, E., \& Stellatos, G. J. (2008). The impact of full disk encryption on digital forensics. ACM SIGOPS Operating Systems Review, 42(3), 93-98.

Cohan, P. (2013). Four reasons google bought waze. Retrieved 6.16.2015, from http://www.forbes.com/sites/ petercohan/2013/06/11/four-reasons -for-google-to-buy-waze/

Colombini, C. M., Colella, A., Castiglione, A., \& Scognamiglio, V. (2012). The digital profiling techniques applied to the analysis of a gps navigation device. In Innovative mobile and internet services in ubiquitous computing (imis), 2012 sixth international conference on (pp. 591-596).

Distefano, A., Me, G., \& Pace, F. (2010). Android anti-forensics through a local paradigm. digital investigation, 7, S83S94.

Hoog, A. (2011). Android forensics: investigation, analysis and mobile security for google android. Elsevier.

Hoog, A., \& Strzempka, K. (2011). iphone and ios forensics: Investigation, analysis and mobile security for apple iphone, ipad and ios devices. Elsevier.

Karpisek, F., Baggili, I., \& Breitinger, F. (2015). Whatsapp network forensics: Decrypting and understanding the whatsapp call signaling messages. Digital Investigation, 15, 110-118.

Kent, K., Chevalier, S., Grance, T., \& Dang, H. (2006). Guide to integrating forensic techniques into incident response. NIST Special Publication, 800-86.

Last, D. (2009). Gps forensics, crime, and jamming. GPS World.

Leber, J. (2013). A shrinking garmin navigates the smartphone storm. Mar.

Leppert, S. (2012). Android memory dump analysis. Student Research Paper, Chair of Computer Science, 1.

Lessard, J., \& Kessler, G. (2010). Android forensics: Simplifying cell phone examinations.

Macht, H. (2013). Live memory forensics on android with volatility. Friedrich-Alexander University Erlangen-Nuremberg.

Mahajan, A., Dahiya, M., \& Sanghvi, H. (2013). Forensic analysis of instant messenger applications on android devices. arXiv preprint arXiv:1304.4915.

Maus, S., Höfken, H., \& Schuba, M. (2011). 
Forensic analysis of geodata in android smartphones. In International conference on cybercrime, security and digital forensics, http://www. schuba. fh-aachen. de/papers/11-cyberforensics. pdf.

McMillan, J. E. R., Glisson, W. B., \& Bromby, M. (2013). Investigating the increase in mobile phone evidence in criminal activities. In System sciences (hicss), 201346 th hawaii international conference on (pp. 4900-4909).

Morrissey, S., \& Campbell, T. (2010). ios forensic analysis for iphone, ipad, and ipod touch (Vol. 23). Springer.

Nutter, B. (2008). Pinpointing tomtom location records: A forensic analysis. Digital Investigation, 5(1), 10-18.

Simão, A. M. d. L., Sícoli, F. C., Melo, L. P. d., Deus, F. E. G. d., \& Sousa Júnior, R. T. d. (2011). Acquisition and analysis of digital evidencein android smartphones.

Statista. (2012). People who use their cell phone for maps/gps navigation (usa), 2012. Retrieved 2015-9-29, from http:// www.statista.com/statistics/231615/ people-who-use-their-cell-phone-for -maps-gps-navigation-usa/

Statista. (2015). Mobile apps: U.s. smartphone audience reach 2015 - statistic. Retrieved 2015-9-29, from http://www.statista.com/ statistics/281605/reach-of-leading -us-smartphone-apps/

Statista. (2016). Number of smartphone users $^{*}$ worldwide from 2014 to 2019 (in millions). Retrieved 20.1.2016, from http://www.statista.com/statistics/ 330695/number-of-smartphone-users -worldwide/

Storozuk, D. (2014). Waze forensics a digital forensic capstone research project. Retrieved 7.6.2015, from http://wazeforensics.blogspot.com/

Strawn, C. (2009). Expanding the potential for gps evidence acquisition. Small Scale Digital Device Forensics Journal, 3(1), 1-12.

Sylve, J., Case, A., Marziale, L., \& Richard, G. G. (2012). Acquisition and analysis of volatile memory from android devices. Digital Investigation, 8(3), 175-184.

Thing, V. L., Ng, K.-Y., \& Chang, E.-C. (2010). Live memory forensics of mobile phones. digital investigation, 7, S74-S82.

Tso, Y.-C., Wang, S.-J., Huang, C.-T., \& Wang, W.-J. (2012). iphone social networking for evidence investigations using itunes forensics. In Proceedings of the 6th international conference on ubiquitous information management and communication (p. 62).

Umale, M. M. N., Deshmukh, A., \& Tambhakhe, M. (2014). Mobile phone forensics challenges and tools classification: A review. International Journal on Recent and Innovation Trends in Computing and Communication, 2(3), 622-626.

Van Eijk, O., \& Roeloffs, M. (2010). Forensic acquisition and analysis of the random access memory of tomtom gps navigation systems. Digital Investigation, 6(3), 179188.

Vidas, T., Zhang, C., \& Christin, N. (2011). Toward a general collection methodology for android devices. digital investigation, 8, S14-S24.

Walnycky, D., Baggili, I., Marrington, A., Moore, J., \& Breitinger, F. (2015). Network and device forensic analysis of android social-messaging applications. Digital Investigation, 14, S77-S84. 
Table 5: Android - summary of results

\begin{tabular}{|c|c|c|}
\hline Application & File & Description \\
\hline Google Maps & da_destination_history & $\begin{array}{l}\text { SQLite database that contained } \\
\text { data related to navigations that oc- } \\
\text { curred within Google Maps }\end{array}$ \\
\hline Google Maps & search_history.db & $\begin{array}{l}\text { SQLite database that contained } \\
\text { data related to searches that oc- } \\
\text { curred within Google Maps }\end{array}$ \\
\hline Google Maps & DATA_SYNC_DATA & $\begin{array}{l}\text { Contained data from the user's ac- } \\
\text { count that did not take place on the } \\
\text { given device or took place prior to } \\
\text { Google Maps being uninstalled }\end{array}$ \\
\hline Waze & waze_log.txt & $\begin{array}{l}\text { Text file that contained location } \\
\text { data related to routes selected by } \\
\text { the user and their current locations }\end{array}$ \\
\hline Waze & user.db & $\begin{array}{l}\text { SQLite database that contained } \\
\text { data related to navigations selected } \\
\text { by the user }\end{array}$ \\
\hline MapQuest & search.db & $\begin{array}{l}\text { SQLite database that contained } \\
\text { data related to the navigation his- } \\
\text { tory of the user }\end{array}$ \\
\hline Bing & SearchHistoryDatabase & $\begin{array}{l}\text { OSQLite database that contained } \\
\text { user search history when private } \\
\text { browsing was disabled }\end{array}$ \\
\hline Scout & scoutAppDatabase.db & $\begin{array}{l}\text { SQLite database that contained } \\
\text { navigation and messaging data re- } \\
\text { lated to the user }\end{array}$ \\
\hline Scout & Route $_{-}^{*}$ & $\begin{array}{l}\text { XML file that contained the differ- } \\
\text { ent routes for navigation sessions, } \\
\text { the * represented the unix times- } \\
\text { tamp of the start of the respective } \\
\text { navigation session }\end{array}$ \\
\hline
\end{tabular}


Table 6: iOS - summary of results

\begin{tabular}{|c|c|c|}
\hline Application & File & Description \\
\hline Apple Maps & GeoHistory.mapsdata & $\begin{array}{l}\text { Plist file that contained data related } \\
\text { to the user's search history }\end{array}$ \\
\hline Waze & user.db & $\begin{array}{l}\text { SQLite database that contained } \\
\text { data related to navigations selected } \\
\text { by the user }\end{array}$ \\
\hline MapQuest & com.aol.mapquest.plist & $\begin{array}{l}\text { Plist file that contained navigation } \\
\text { data relating to the user }\end{array}$ \\
\hline Bing & Historys.plist & $\begin{array}{l}\text { Plist file that contained data that } \\
\text { pertained to user searches }\end{array}$ \\
\hline Bing & *.dat (Non-private browsing) & $\begin{array}{l}\text { Plist file that contained data on the } \\
\text { most recent search, the }{ }^{*} \text { repre- } \\
\text { sented an integer value }\end{array}$ \\
\hline Bing & *.dat (Private browsing) & $\begin{array}{l}\text { Plist file that contained data on the } \\
\text { most recent private search, the * } \\
\text { represented an integer value }\end{array}$ \\
\hline Scout & com.telenav.scout.pushstore.log & $\begin{array}{l}\text { Plist file that contained incomplete } \\
\text { navigational data }\end{array}$ \\
\hline
\end{tabular}


